\title{
Recent developments with gaseous detonation drivers for a shock tunnel
}

\author{
H-r Yu \\ Laboratory of High Temperature Gas Dynamics, Institute of Mechanics, \\ Chinese Academy of Sciences, Beijing 100080, China
}

\begin{abstract}
Early researches on the detonation driven shock tube are reviewed briefly. Calculation results demonstrated that an improvement in attenuation of incident shock wave generated by forward driver can be obtained, provided the diameter of the driver is larger than that of the driven section and an abrupt reduction of cross-section area is placed just beyond the diaphragm and were verified by the experiments. Inserting a cavity ring between the driver and the driven section can make the flow field parameters behind a detonation rather uniform. An additional backward-detonation driver was proposed to attach to primary forward-detonation driver. The Taylor wave in the primary driver can be eliminated completely, if the ratios of initial pressure in additional driver to that in primary driver exceed the threshold value (about 7 times).
\end{abstract}

\section{Introduction}

In view of the enthalpy and the pressure requirements for hypervelocity test, the shock tunnel must incorporate a high performance driver. Among the exiting driving techniques, only a few of these meet the requirements of the high performance driver. The detonation drivers are capable of producing high enthalpy and high pressure in the meantime. Besides they appear easier to operate and have a lower capital investment. Before a description recent developments with detonation drivers, a brief summary of early research will be presented.

\section{Early research}

In the processes of experiment on combustion driver, Hertzberg \& Smith (1954) unexpectedly found that Shock Mach numbers were produced which were greater than those calculated on the assumption of normal shock tube flow and constant volume combustion. Gerard attributed the phenomenon to detonation taking place in the driver. Bird (1957) analyzed subsequently wave processes in detonation driver.

For practical purpose, two sites of detonation initiation are feasible, namely the backward- and forward-detonation driver modes. In the backward-detonation driver, the detonation wave is initiated at the main diaphragm, and its propagation direction is opposite to that of the incident shock wave. In the forward-detonation driver, the detonation wave is initiated at the breech end of the driver, and its moving direction is the same as the incident shock wave propagation.

$\mathrm{Yu}$ (1963) and Lee (1967) performed the experiments on backward-detonation driver. Yu's results indicated that the strength of shock wave generated by backward-detonation driver is stronger than that by combustion driver, which is different from Bird's prediction, because Bird did not consider the effect of wall heat transfer on combustion or detonation. The propagation speed of flame is much slower than that of detonation wave, 
so that the heat loss of the former is greater than that of the latter. The attenuation of shock wave caused by backward-detonation driver is very weak and the result exhibited good repeatability. Nevertheless, the reflection of the detonation produces considerable overpressure on the breech end of the tube so that the allowable initial pressure is restricted within low pressure scope. Hence, the backward-detonation driver do not meet the practical requirements of hypervelocity testing facilities.

Waldron(1958) examined experimentally the driving behavior of forward-detonation driver. He came to the conclusion that shock waves generated by a forward-running detonation wave attenuate substantially both with length of shock propagation and with time because of the decaying flow field behind the detonation and were unacceptable for use in aerodynamic testing facilities. Balcerzak and Johnson (1965) applied the forwarddetonation driver to simulation of blast waves in which the shock attenuation is unimportant.

Coats and Gaydon (1965) used a double driver with unheated hydrogen as the additional driver gas and oxy-hydrogen mixture in primary driver. They expected to perform two operating modes. The first mode is that, when the diaphragm between the additional and the primary detonation driver bursts, a strong shock wave is produced in the detonable mixture, which then detonates rapidly. The second mode is that the bursting of the diaphragm shall produce a rather weaker shock in the detonable mixture, so that this does not detonate in the weaker shock, but in the shock reflected from the main diaphragm (between the detonation driver and a driven section). The experimental observations show that the objective of the first mode did not be completed, but the second mode (i.e. detonation initiation by reflected shock) had been realized. Gier and Jones (1969) realized similarly the detonation initiation by the reflected shock.

\section{Method for reducing the mechanical loading of backward-detonation driver}

In the backward-detonation driver, the detonation wave is initialed at main diaphragm and propagates upstream to the breech end of the driver. When the detonation reaches the end plate, the high pressure over hundred times of the initial pressure arises from the reflection of the detonation.

Yu $(1989,1992)$ proposed to attach a buffer tube to the breech end of the driver tube for mitigating the excessive reflected pressure. When the detonation front reaches the light diaphragm between the buffer and detonation tube, it will be break immediately open. Because the initial pressure of buffer tube is much lower in comparison with that of the detonation driver, the reflected wave will be a rarefaction wave instead a shock wave. Thus the high reflected pressure disappears at the breech end of the backward-detonation driver. It makes high initial pressure of detonable mixture can be practically used.

The high reflected pressure may be transferred onto the end of the buffer tube. The flow process in the buffer tube is equivalent to that in the driven section of normal shock tube. According to the theory of shock tube flow, it is well known that the pressure behind reflected shock wave is steadily decreasing along with the drop of the initial pressure in driven section on condition of the same of other initial parameters. Therefore, the initial pressure in the buffer tube is the lower the better. Fig. 1 shows the experimental results of relations between the reflected pressure peak on the end wall and the initial pressure in the buffer tube. (Yu and Zhao, 1996) 


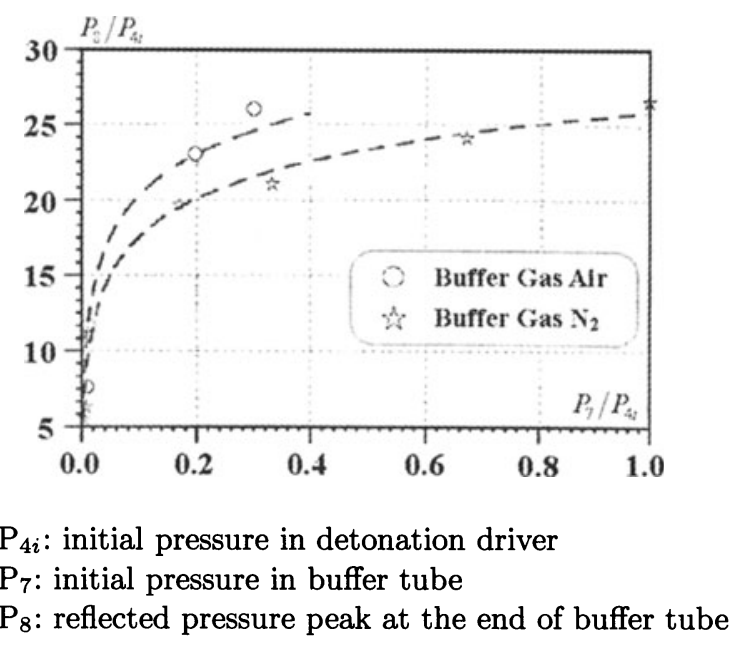

Fig. 1. reflected peak pressure vs initial pressure in buffer tube

The comparison between the driving capability of the forward-detonation driver and that of the backward-detonation driver shows that the former is much stronger than that of the latter. Because the effective state parameters of the forward-detonation driver are higher than that of the backward-detonation driver, especially the detonation product generated in the forward-detonation driver possesses the tremendous kinetic energy in addition. If the detrimental effect of the Taylor wave in a forward-detonation driven shock tube can be mitigated to the extent of practical feasibility or the Taylor wave itself can be eliminated, the better choice for hypervelocity testing facilities may be the forward-detonation tunnel.

\section{Methods for mitigating detrimental effect of Taylor wave}

Several conceptions were proposed to mitigating the detrimental effect of Taylor wave.

\subsection{Lengthening the driver}

The flow field produced by detonation wave in a closed tube consists of a constant-velocity detonation itself followed a self-similar Taylor wave that bring the post-detonation gas set in motion back to rest (Taylor, 1950). The length of the pressure, temperature and velocity drop region is about one-half of the distance to which the detonation has propagated. The longer this distance, the smaller the pressure, temperature and velocity drop per unit length because the total drops is fixed. This means that a longer detonation driver can provide a more uniform driving flow behind the detonation wave. However, the effectiveness attained in the method is limited and often obtained at the expense of excessive consumption of the detonable mixture.

\subsection{Increasing diameter of the driver}

The Mach numbers of incident shock wave can be increased by using a driver with a reduction in cross-section area just beyond the main diaphragm (Alpher and White, 
1958). In the case of using a detonation, when the detonation wave initiated at the breech end of the driver reaches the converging section, there will form a reflected shock wave, which will interact with the opposing rarefaction wave (Fig.2). The rarefaction fan will be expanded and the decay of state parameters in flow is reduced. In the meantime, the gas in outer ring fills continuously in main flow from lateral side, which makes further the flow field uniform. Thus the attenuation of incident shock wave can be weakened substantially. The experimental results had verified the conception (Yu, 1999).

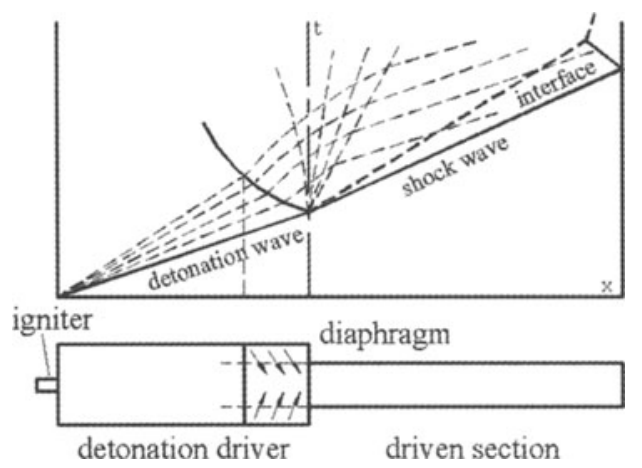

Fig. 2. Diagram of flow in variable cross-section shock tube with forward detonation driver

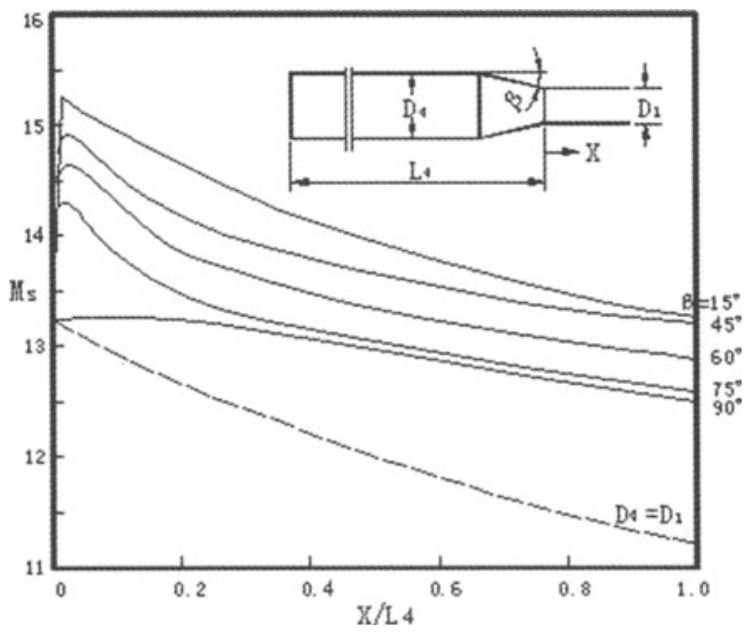

Fig. 3. Variations of Mach number of incident shock wave

The computation study on the behavior of a variable cross-section forward-detonation driven shock tube have completed by Yang (2000). The calculation results (Fig.3) show that the half conic angles of converging section dominate the attenuation of incident shock wave along the driven section. The maximum Mach number of incident shock wave generated by the variable cross-section shock tube is identical with that by the uniform shock tube, but the shock attenuation of former is much weaker than the latter, 
if the half conic angle of converging section is equal to $90^{\circ}$. In the range of half conic angle $\beta \leq 75^{\circ}$ the maximum Mach number and the shock attenuations are steadily raising along with the decrease of the half conic $\beta$ angle. The optimum half conic angle $\beta$ should choose $90^{\circ}$.

\subsection{Inserting a cavity ring}

Effects of variable cross-section area of detonation tube on the uniformity of flows followed the detonation wave were numerically examined by Jiang et al. (1999). It is found that the flow field behind a detonation wave generated by the tube with a cavity ring is more uniform than that by the tube with a $30^{\circ}$ or $45^{\circ}$ converging section. In order to verify the results, a forward-detonation driven shock tube has been constructed (Jiang et al. 2002). Its detonation driver is composed of a big tube $(3.87 \mathrm{~m}$ long and $90 \mathrm{~mm}$ in diameter), a cavity ring $(130 \mathrm{~mm}$ in diameter) and a small tube $(180 \mathrm{~mm}$ long and $60 \mathrm{~mm}$ in diameter) in the order. The driven section is $7 \mathrm{~m}$ long and $60 \mathrm{~mm}$ in diameter. According to the measured reflected pressure, the driver with the cavity ring $(360 \mathrm{~mm}$ long) can generated uniform test gas.

\section{Method of eliminating the Taylor rarefaction wave}

If a moving piston is following a detonation wave and its velocity reaches or exceeds C-J value of the detonation product, the Taylor wave will disappear. Moreover, what follows the detonation wave will be a gas column with constant velocity and uniform state parameters. Because the velocity of detonation product is very high, it is difficult to accelerate a mechanical piston to such high speed in a shock tube. Coats and Gaydon (1965) employed first an additional driver utilizing hydrogen as the driving gas attached to the upstream end of the detonation driver, which generated a moving gaseous piston instead of a traveling mechanical piston. Bakos et al. (1996) took helium as driving gas in the additional driver. They employed the pressure of unheated hydrogen or helium are all rather lower, therefore the Taylor wave can not be eliminated completely and the initial strength of the shock in detonable mixture is not strong enough to direct initiate the detonation wave reliably.

For equaling the velocity and pressure of the gaseous piston to that of the detonation product respectively, the initial pressure unheated hydrogen or helium should exceed hundred times higher than that of detonable mixture in the detonation section. The initial pressure of detonable mixture in the driver of practical shock tunnel is most often several MPa or more. Therefore, the required initial pressure of hydrogen or helium must be excessively high in order to eliminate the Taylor wave completely. Not only are the required high-pressure pump and vessel equipment too expensive, but also the structures of the additional driver as well as the rupture technology of thick diaphragm present severe technical problem.

For solving these technical problems, a backward-detonation driver utilizing the same detonable mixture in the primary detonation driver is employed as the additional driver instead of the light gas drivers. Under these conditions, the initial pressure of the additional driver need only be several times higher than that of the primary driver for eliminating the Taylor wave completely. In addition, the post-detonation gas ejected from the additional driver can directly initiate a detonation wave in the primary driver. Thus, another technical impediment is also solved in practice. 


\subsection{Estimation of the critical ratio of initial pressure}

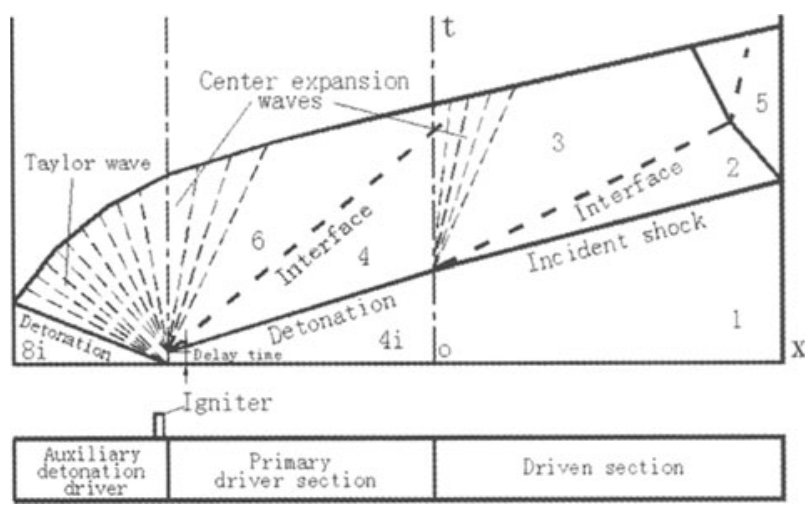

Fig. 4. Wave diagram of shock tube with double detonation drivers

The double detonation driven shock tube, shown schematically in Fig.4, consists of both the additional backward-detonation driver and the primary forward-detonation driver in which the same oxy-hydrogen mixture are utilized and a driven section. The post-detonation gases in the additional driver penetrating the Taylor wave are decelerated to the stationary state and then accelerated to the $\mathrm{u}_{6}$ after penetrating the central rarefaction wave caused by the rupture of the main diaphragm. According relation of simple wave

$$
u_{C J}+\frac{2}{(\gamma-1)} a_{C J}=u_{6}+\frac{2}{(\gamma-1)} a_{6}
$$

On the assumption that (1) the component ratio of hydrogen to oxygen is 3 , (2) the initial temperature of oxy-hydrogen is $18^{\circ} \mathrm{C},(3)$ the initial pressure has no influence on the pressure and acoustic speed ratios of the wave front to wave rear, (4) the special heat ratio of post-detonation gases in the expansion process is constant $(\gamma=1.2),(5)$ the Taylor wave following the primary detonation is just eliminated completely, we obtained

$$
\begin{aligned}
& u_{6}=u_{4}=u_{C J} \\
& a_{6}=a_{C J}-(\gamma-1) u_{C J}
\end{aligned}
$$

The values taken from Edwards et al.(1959) $\left[u_{C J}=1.4 \times 10^{3} \mathrm{~m} / \mathrm{s}, a_{C J}=1.8 \times 10^{3} \mathrm{~m} / \mathrm{s}\right]$

$$
a_{6}=1.52 \times 10^{3} \mathrm{~m} / \mathrm{s}
$$

The critical initial pressure ratio between the additional and the primary detonation driver

$$
\frac{P_{8 i}}{P_{4 i}}=\frac{\left(P_{C J}\right)_{a}}{\left(P_{C J}\right)_{p}}=\left(\frac{a_{C J}}{a_{6}}\right)^{\frac{2 \gamma}{\gamma-1}}=7.6
$$

The corresponding critical initial pressure ratios of unheated light gas are about 100 times $\left(\mathrm{H}_{2}\right)$ and 450 times $(\mathrm{He})$ respectively. 
If ratios of initial pressure exceed the critical value, an over-driving detonation wave will be formed in the primary driver, velocity, acoustic speed and pressure of the detonation product are increased also, and thus the driving capability is steadily strengthen along with further increasing the ratio of the initial pressure.

\subsection{Experimental results}

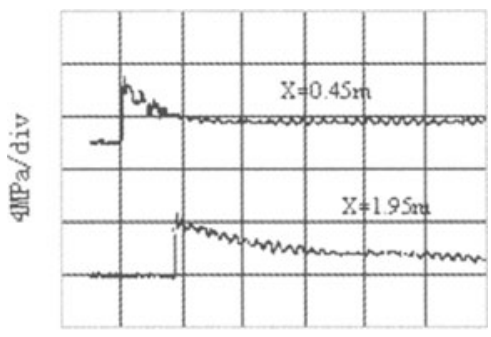

$0.5 \mathrm{~ns} / \mathrm{div}$

(a) Single detoration section $\left(\mathrm{P}_{4}=0.25 \mathrm{MPa}\right)$

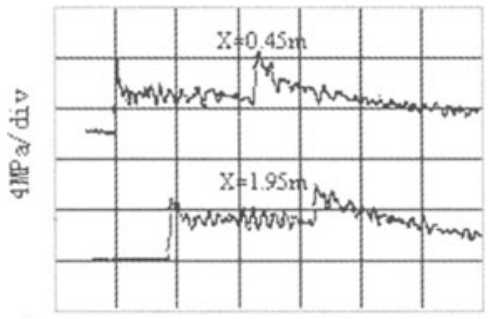

$0.5 \mathrm{~ns} / \mathrm{div}$

(b) Double detonation section

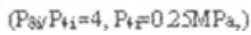

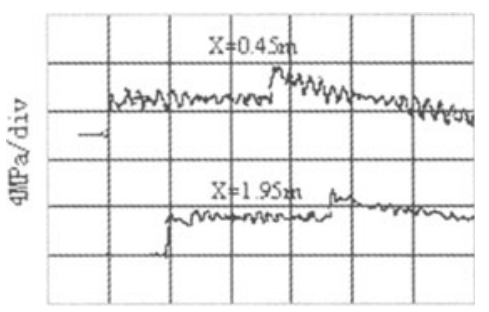

$0.5 \mathrm{~ms} / \mathrm{div}$

(c) Double defonation section $\left(\mathrm{P}_{2 /} \mathrm{P}_{4 \mathrm{i}}=6, \mathrm{P}_{4}=02 \mathrm{MPa}\right)$

Fig. 5. Pressure-time curves in the primary driver

The measured variations of pressure with time at different sites in the primary detonation driver are depicted in Fig.5. For comparison, the results of single forward-detonation driver were included and were shown in Fig.5a, in which the pressure raised instantaneously to be approximate eighteen times higher than the initial value due to the arrival of detonation wave. Subsequently, the pressure gradually dropped below half of the peak value, owing to the effect of the Taylor wave, and then maintained a constant value. The measured results of double detonation driver were shown in Figs.5b and 5c. In Fig.5b, the pressure rose abruptly and then dropped also. However, the period of the drop was shorter and pressure platform value was higher than that of single detonation driver, because the initial pressure ratio $\left(\mathrm{P}_{8 i} / \mathrm{P}_{4 i}=4\right)$ was lower than the critical value, the Taylor wave was only eliminated partly. Fig.5c reveals the pressure-time curve for the condition of $\mathrm{P}_{8 i} / \mathrm{P}_{4 i}=6$. Because the initial pressure ratio was close to the critical value, the pressure rose, but then did not drop. This response indicates that the Taylor wave was eliminated. The pressure at latter half of the curve had an upward jump, which was 
caused by the overtake of the reflected shock from the tail-end wall of the additional driver. Such interference will disappear if the length of each section is designed properly.

Under the condition of the same of initial pressures and of the maximal Mach numbers in the driver section, the comparison of the Mach number variations of the incident shock wave generated by double detonation drivers with those caused by single forward detonation driver are shown in Fig.6. It shows that the attenuation of an incident shock wave generated in the double drivers is weaker than that by a single detonation driver. However, the attenuation of incident shock was appreciable, even for a wave generated in the double detonation driver because the attenuation was dependent not only the quality of the driver gas, but also on boundary layer of the driver section wall. For the apparatus used in our work, the surface state of the tube wall was not ideal and the initial pressure in driven section was lower, inevitably boundary layer effect induced substantial attenuation of the incident shock wave. In practical applications, we can improve the state of the tube wall and increase the initial pressure, thus the boundary layer effect will decrease considerably and the attenuation of the incident shock wave generated by the double detonation driver will be reduced significantly.

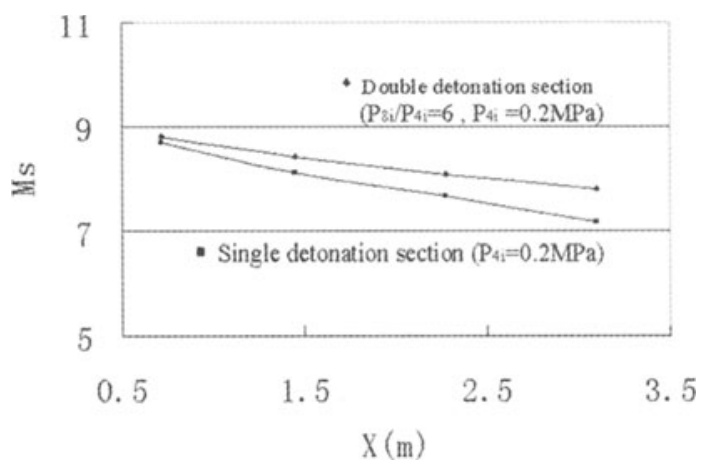

Fig. 6. Attenuation characteristics of incident shock wave

\section{Conclusions}

The driving capability of the forward-detonation driver is much higher than that of the backward-detonation driver. Only either the rarefaction fan following detonation wave is eliminated completely or its detrimental influence is mitigated to the extent of practical feasibility, the forward-detonation can be practically applied to aerodynamic testing facilities. The double detonation drivers with the same oxy-hydrogen mixture as a detonable gas were proposed to eliminate the Taylor wave in the primary detonation driver. When the diaphragm separating the additional and the primary driver bursts, an overdriving detonation wave is produced. The pressure and temperature profile as well as velocity distribution behind the detonation is substantial flat, namely the Taylor wave is eliminated completely provided that the ratios of initial pressure in additional driver to that in primary driver exceed the threshold value. Furthermore, the state parameters and the velocity of post-detonation gas are steadily increasing along with the increasing ratio 
of initial pressure. Consequently, it constitutes a new way to further raise the enthalpy of the test flow.

Lengthening the driver, increasing diameter of the driver and inserting a cavity ring can all mitigating the detrimental influence of the Taylor wave to some extent. In the variable cross-section shock tube, using a driver tube with an abrupt reduction in crosssection area just beyond the diaphragm can obtain substantially good results.

\section{Acknowledgement}

I express my cordial thanks to Prof. Grönig \& Dr. F. Zhang who kindly supported me to start preliminary experiment for overcoming the performance defects of detonation drivers in Aachen Shock Wave Laboratory. Thanks go also to a number of students and colleagues of the Key Laboratory of High Temperature Gas Dynamics, CAS. Especially Mr. H. Chen, H. Feng \& J.M. Lin, Dr. W. Zhao.

This work was supported by National Foundation of Nature Science of China (10082004).

\section{References}

1. R.A. Alpher, D.R. White: J. Fluid Mechanics 3, 457 (1958)

2. R.J. Bakos et al.: AIAA 96-2193, (1996)

3. M. Balcargak, M.R. Johnson: In: Proc.5th.Intern. Shock Tube Symp., (1996) pp. 1111

4. G.A. Bird: AGARD Rep 146, (1957)

5. P.B. Coates, F.R.S. Gaydon: Proc. Roy. Soc. London A283, 18 (1965)

6. D.H. Edwards, G.T. Williams, J.C. Breeze: J. Fluid Mechanics 4, 497 (1959)

7. H.L. Gier, T.G. Jones: In: Proc.7th.Intern. Shock Tube Symp., (1969) pp. 272

8. A. Hertzberg, W.E. Smith: J. Appl. Phys 25, 130 (1954)

9. Z. Jiang et al.: In: Proc. 22nd ISSW, (1999)

10. Z. Jiang et al.: J. AIAA 10, 2009 (2002)

11. B.H.K.L. Lee: J. AIAA 5, 791 (1967)

12. G.I. Taylor: Proc. Roy. Soc. A200, 234 (1950)

13. H.F. Waldron: UTIA Rep 50 (1958)

14. H.W. Yang: Docter Thesis, Beijing University, China (2000)

15. H-r Yu: Thesis, Institute of Mechanics, Chinese Academy of Sciences, Chinese (1963)

16. H-r Yu: In: Proc.1989 Japan Symp. on Shock Wave Phenomena, (1989) pp. 1

17. H-r Yu, Esser B, Lenartz M \& Groenig H: Shock Waves, 4, 245 (1992)

18. H-r Yu: Acta Mechanica Sinica 2, 97 (1999)

19. H-r Yu, W. Zhao: In: Proc.20th. Intern. Symp. on Rarefected Gasdynamic, (1996) pp. 927 